\title{
Factores que influyen en la compra de alimentos orgánicos en México. Un análisis mixto
}

\section{Factors Influencing the Purchase of Organic Food in Mexico. A Mixed Analysis}

\author{
Gloria Lety Lopez Salazar ${ }^{\mathrm{a}, *} \square$ \\ a) Departamento de Finanzas y Administración, Universidad de Guanajuato, Mexico \\ * Corresponding author: gletylopez11@gmail.com (Gloria Lety Lopez Salazar)
}

\begin{abstract}
Resumen
El objetivo de este trabajo de investigación es analizar la demanda de alimentos orgánicos y determinar los factores que influyen en la disposición a pagar un sobreprecio por alimentos orgánicos en el mercado mexicano. Se diseñó y validó un cuestionario para estudiar el comportamiento de compra de 270 consumidores de la ciudad de Celaya, Guanajauto, quienes pertenecen a uno de los tres niveles socioecónomicos más privilegiados del país; así mismo, para profundizar en el tema se llevaron a cabo sesiones de grupo. Los resultados muestran que la conciencia de beneficios de los productos orgánicos, la edad del consumidor y la disponibilidad del producto son factores que influyen la disposición a pagar un sobreprecio por alimentos orgánicos.
\end{abstract}

Palabras clave: decisión de compra; alimentos orgánicos; disposición a pagar

Clasificación JEL: D87; L25; M13; M39; Y20

\section{Abstract}

The objective of this research is to analyze the factors influencing the willingness to pay extra for organic food and to determine its demand on the Mexican market. An instrument was designed and validated in order to study the purchasing behavior of 270 residents of Celaya City (State: Guanajuato). Target market: people who belongs to the top 3 socioeconomic levels in the Country. The findings show that the factors that influence the willingness to pay extra for organic food are items such as the consumer age, the level of awareness on the organic food benefits and the availability of the products.

Keywords: purchase decision; organic food; willingness to pay

JEL Classification: D87; L25; M13; M39; Y20 


\section{Introducción}

Desde hace más de dos décadas la demanda de productos orgánicos ha experimentado altas tasas de crecimiento a nivel mundial destinando 58 millones de hectáreas y generando 90 mil millones de dólares de facturación (Willer, Lernoud y Kemper, 2018). Esta tendencia positiva caracteriza también al mercado mexicano pues ha logrado posicionarse como el segundo productor a nivel mundial de vegetales orgánicos con la participación de 210 mil productores registrados, el cuarto productor de alimentos orgánicos en América y el séptimo a nivel mundial (Proméxico, 2017). Para el año 2020, se espera que el mercado de alimentos y bebidas orgánicas en México mantenga una tasa de crecimiento promedio anual del 8.4\% (Global Organic Trade Guide, -Guía de comercio global orgánico- 2017).

Actualmente el $85 \%$ de la producción orgánica certificada se exporta, principalmente a países como Estados Unidos, Alemania, Francia, Canadá, Japón y Reino Unido (Global Organic Trade Guide, 2017). Grupo Herdez SAB de CV concentra el 34.5\% de las ventas de productos orgánicos, seguido por Nestlé con el 5.2\% y por el Grupo Industrial Cuadritos Biotek SA de CV con el 4.6\% (Global Organic Trade Guide, 2017). La actual demanda internacional de alimentos orgánicos producidos en México tiene su origen en el interés que tuvieron los países desarrollados en obtener alimentos orgánicos producidos con técnicas tradicionales para conservar sus características naturales. Posteriormente las mismas empresas extranjeras ofrecieron los apoyos necesarios para que los productores mexicanos transitaran al cultivo de productos orgánicos (Gómez, Gómez y Schwentesius, 2002).

A pesar de que en México se produce un alto porcentaje de alimentos orgánicos a nivel mundial, no representa uno de sus principales consumidores ya que sólo el $15 \%$ de los alimentos orgánicos que produce son para consumo doméstico. Estados Unidos consume el $40.6 \%$ de los productos orgánicos a nivel mundial y, en conjunto, los países europeos demandan el $58 \%$ de ellos, lo cual implica que solo el 1.4\% del consumo mundial se encuentra en el resto de los países (Global Organic Trade Guide, 2017). Estos datos son un indicio del bajo nivel de demanda que tienen los alimentos orgánicos en México en comparación a otros países como Alemania, Japón, Francia, Italia, Gran Bretaña, Canadá y Suiza. Entonces, ¿Qué está pasando con el mercado doméstico en México? ¿Por qué ha sido tan limitado el consumo de productos orgánicos? ¿Qué factores han influido en su consumo?

La falta de una cultura alimentaria sana, los altos precios y el grado de disponibilidad de los productos orgánicos son factores que limitan su demanda. De acuerdo a la Procuraduría Federal del Consumidor (PROFECO) los productos orgánicos en México tienen un sobreprecio de entre $11 \%$ y $337 \%$ lo cual representa una gran desventaja para el mercado de los alimentos orgánicos, sobre todo porque son pequeños productores campesinos e indígenas quienes en su mayoría han adoptado esta forma de producción (Schwentesius, Gómez, Ortigoza y Gómez, 2014).

Por lo tanto, el objetivo de este trabajo de investigación es caracterizar el mercado de los alimentos orgánicos en México para acelerar su índice de consumo, pues aún es incipiente lo que se ha estudiado de ellos. Así mismo, se tiene como objetivo analizar los factores que influyen en la disposición a pagar un sobreprecio por alimentos orgánicos, al ser el precio un factor de gran relevancia.

El trabajo está organizado en cuatro secciones. En la primera se realiza una revisión de la literatura sobre el mercado de los alimentos orgánicos y sobre la decisión de compra del consumidor. En la segunda sección se muestra la metodología utilizada en el desarrollo del estudio. Posteriormente se presentan los resultados encontrados y se realiza una discusión sobre ellos. Finalmente, se generan conclusiones, implicaciones y futuros estudios de investigación. 


\section{Revisión de la literatura}

\subsection{Definición de producto orgánico}

El término orgánico hace referencia a aquellos alimentos naturales y saludables que provienen de un nuevo sistema de producción alimenticio que elimina grandes problemas de salud a la población. De acuerdo a la PROFECO los alimentos orgánicos son

"productos que se cultivan, crían y procesan utilizando métodos naturales. En el caso de la agricultura, no se utilizan químicos, como pesticidas, fertilizantes sintéticos, aguas residuales, o variedades transgénicas. En la ganadería no se le administran a las animales hormonas de crecimiento, anabólicos o antibióticos, ni se les alimentan con comida sintética" (PROFECO, 2018, p.1).

Amador (2005) explica que los productos orgánicos son resultado de procesos agrícolas comprometidos con la salud de los consumidores y la conservación del medio; no se utilizan insecticidas, herbicidas ni fertilizantes químicos para su producción, menos aún se consiente el uso de organismos genéticamente modificados (OGMs) vegetales o animales, - y el riego con aguas negras; tampoco la utilización radiaciones con el fin de eliminar gérmenes que pudieran ser dañinos. Por otro lado, con relación a la producción de carne, no está permitido el alimentar a los animales con productos sintéticos y mucho menos aplicarles hormonas con tal de favorecer su crecimiento.

Esta perspectiva es coincidente con la definición de la Organización de las Naciones Unidas para la Alimentación y la Agricultura (ONUAA) quien afirma que la agricultura orgánica está enfocada en "tomar en cuenta las posibles repercusiones ambientales y sociales eliminando la utilización de insumos, como fertilizantes y plaguicidas sintéticos, medicamentos veterinarios, semillas y especies modificadas genéticamente, conservadores, aditivos eirradiación" (ONUAA, 2019). En este sentido, la ONUAA incorpora una visión más amplia sobre el término orgánico ya que toma en cuenta, además de los efectos en la salud, el cuidado del medio ambiente. Cuando se tiene una agricultura orgánica se conserva e incrementa la fertilidad del suelo en el largo plazo, se evitan plagas en los cultivos, disminuye la probabilidad de contaminar el agua subterránea, mitiga el efecto invernadero y el calentamiento global del planeta y se protege la biodiversidad.

Para otros organismos, el término orgánico hace referencia también a un sistema justo de comercialización donde las condiciones laborales y los salarios son justos, no existe explotación laboral infantil y se respetan los derechos de los productores más desfavorecidos (PROFECO, 2018)

En conclusión, la producción orgánica ofrece diversas ventajas como son la producción sin utilización de agroquímicos, la conservación de la fertilidad del suelo, el uso sostenible del suelo, el cuidado del medio ambiente, el uso de conocimientos tradicionales, el uso de policultivos y la sostenibilidad productiva.

\subsection{Certificaciones}

Con el objetivo de garantizar la calidad de los alimentos orgánicos se crearon organismos para avalar que los alimentos están libres de fertilizantes, insecticidas, pesticidas, aguas negras, transformación genética y radiación ionizante (González, 2017).

De acuerdo con la SAGARPA existen catorce organismos nacionales e internacionales que certifican la producción orgánica en México, los cuales son: Agricert México, S.A. de C.V., México Certificadora Orgánica, A.C., Transcanada Organic Certification Services México, S.C., Organic Crop Improvement Association International, A.C., CCOF Servicios de Certificación, S. de R.L. DE C.V., Certificadora Mexicana de Productos y Procesos Ecológicos (Certimex), NSF México, S de R.L. de C.V., Mayacert México, S.C., Metrocert S.A. de C.V. y el Instituto para el Mercado Ecológico, S.A. de C.V., principalmente. En términos generales, los factores que 
éstas consideran en su revisión es el cuidado del agua y del suelo, el procesamiento de los alimentos, las prácticas de cultivo y la responsabilidad social.

En México, la Secretaría de Agricultura y Desarrollo Rural (SADER) es la principal instancia promotora de los productos orgánicos. Esta Secretaría creó el distintivo "Orgánico SAGARPA México" el cual da fe y avala que los alimentos cumplen con los criterios de producción orgánica establecidos en la Ley de Productos Orgánicos, con lo cual se desarrolla la confianza y credibilidad en los consumidores sobre la calidad de los alimentos que consume.

Obtener la certificación implica una fuerte inversión para los productores ya que tienen que financiar la solicitud, proceso de acreditación, impresión y etiquetado del distintivo, así como las cuotas anuales de certificación. Esto representa una fuerte limitante para los pequeños productores ya que no tienen el respaldo económico, obligándolos a comercializar sus productos por otros canales (Amador, 2005).

\subsection{Beneficios de los productos orgánicos}

Son numerosos los beneficios para la salud cuando se consumen alimentos orgánicos ya que al estar libres de conservadores no ponen en riesgo la salud. Las principales ventajas de los orgánicos son:

- Poseen más antioxidantes (alrededor de 30\%), elementos valorados en la salud ya que son auxiliares en la lucha contra enfermedades cardiovasculares y el cáncer.

- Tienen valores hasta tres veces más altos de ciertas vitaminas (como la C), en algunas frutas y verduras.

- Se ha detectado mayor concentración de hierro y nutrientes.

- Tienen menos grasa lo que se traduce en menor riesgo de contraer enfermedades.

- Están recomendados para personas que padecen cáncer, leucemia y diabetes, cuya alimentación debe ser muy particular y rigurosa.

- Son más agradables por sus propiedades organolépticas (sabor, olor, textura).

- La carne orgánica es más magra, lo que implica menos grasa intramuscular y bajos niveles de colesterol.

\subsection{El sobreprecio de los alimentos orgánicos}

Los alimentos orgánicos tienden a ser más caros que los convencionales debido a que tienen una escala menor de producción, requiere de controles estrictos en el proceso productivo, el ciclo de producción es más lento, implica mayores costos de logística, alto costos de certificación y debe tener un empaque especial para conservarlos frescos (Ramírez, 2016; Amador, 2005).

De acuerdo a la PROFECO (2018), los alimentos orgánicos tienen un precio superior a los alimentos convencionales. Productos como la carne de pollo y la harina son productos por los que se paga un mayor sobreprecio, y la leche y quesos por lo que el sobreprecio es mucho menor (entre 11\% y 49\%). Así mismo, el diferencial de precio varía en función de la presentación del producto y del lugar donde se adquiera.

A pesar de que el precio es un factor relevante que ha limitado el incremento de su demanda, los consumidores de alimentos orgánicos no son tan sensibles al precio, más bien están orientados a decidir su compra con base en el valor que perciben del producto (Zielke, 2010).

\subsection{El consumidor orgánico}

Se han desarrollado diversas definiciones del consumidor orgánico con base en el compromiso con la salud y el cuidado del medio ambiente. Sin bien algunas de estas clasificaciones difieren en cuanto a su terminología, son similares en relación a las características que las describen. En la tabla 1 se muestran básicamente tres tipos de consumidores orgánicos: aquellos que solo compran productos saludables y amigables con el medio ambiente; consumidores que 
Tabla 1. Descripción de categorías de consumidores orgánicos y no orgánicos, según diferentes clasificaciones

\begin{tabular}{c|c|c|c}
\hline Hartman Group & NMI & Magrama & $\begin{array}{c}\text { Descripción de categorías de } \\
\text { consumidores }\end{array}$ \\
\hline Consumidores core & Consumidores Lohas & $\begin{array}{c}\text { Consumidores } \\
\text { convencidos }\end{array}$ & $\begin{array}{c}\text { Involucrados e influyentes en la industria } \\
\text { orgánica; preocupados por la salud y por el } \\
\text { planeta. }\end{array}$ \\
\hline $\begin{array}{c}\text { Consumidores de } \\
\text { nivel medio }\end{array}$ & $\begin{array}{c}\text { Consumidores } \\
\text { naturalites }\end{array}$ & $\begin{array}{c}\text { Consumidores } \\
\text { ecologistas }\end{array}$ & $\begin{array}{c}\text { Preocupados por el medio ambiente y por } \\
\text { hábitos de consumo responsable. }\end{array}$ \\
\cline { 2 - 4 } & $\begin{array}{c}\text { Consumidores } \\
\text { preocupados por la } \\
\text { salud }\end{array}$ & $\begin{array}{c}\text { Usuarios frecuentes de productos } \\
\text { naturales y orgánicos preocupados por su } \\
\text { salud personal y, en menor medida, por el } \\
\text { medio ambiente. }\end{array}$ \\
\cline { 2 - 4 } & Consumidores \\
drifters & $\begin{array}{c}\text { Consumidores } \\
\text { desimplicados }\end{array}$ & $\begin{array}{c}\text { Se dejan llevar por las últimas tendencias; } \\
\text { su involucramiento en el movimiento } \\
\text { ambiental es intermitente. }\end{array}$ \\
\hline consumidores & $\begin{array}{c}\text { Consumidores } \\
\text { penvencionales }\end{array}$ & $\begin{array}{c}\text { Consumidores } \\
\text { despreocupados }\end{array}$ & $\begin{array}{c}\text { Más centrados en sí mismo; son prácticos, } \\
\text { racionales y conscientes sobre los } \\
\text { desperdicios, pero privilegian el ahorro. }\end{array}$ \\
\hline
\end{tabular}

Fuente: Higuchi (2015).

de manera cotidiana compran productos orgánicos y limpios principalmente por salud; y consumidores que los adquieren por moda.

Desde un enfoque más psicológico, Schifferstein y Ophuis (1998) definen al consumidor tradicional de productos orgánicos como aquella persona donde "el consumo de alimentos orgánicos forma parte de su modo de vivir. Es resultado de una ideología, conectada a un sistema de valores particular, que afecta las medidas de personalidad, actitudes y su comportamiento de consumo". (p.119)

\subsection{Proceso de decisión de compra}

De acuerdo a Lamb, Hair y McDaniel (2011) el comportamiento del consumidor está vinculado con los procesos de toma decisiones que los consumidores siguen para elegir su compra. De acuerdo al modelo de Schiffman, Lazar y Wisenblit (2010) este proceso consta de tres etapas: entrada, proceso y salida (ver Figura 1). La primer etapa se enfoca a los factores externos que influyen en las decisiones de compra, como son las estrategias de mercadotecnia y el ambiente sociocultural. La segunda etapa hace referencia a la identificación de una necesidad la cual es influenciada por cuestiones psicológicas del consumidor, tales como la motivación, la percepción, la personalidad y las actitudes. Una vez que reconoce su necesidad, el consumidor busca información sobre las opciones que tiene disponibles para cubrir dicha necesidad, evalúa las alternativas identificadas, y es entonces cuando selecciona el producto. La tercera etapa se concentra en la evaluación de la compra, la cual determina la repetición de la venta y la reputación generada del producto.

En este mismo sentido, Viera, Gálvez y Navarro (2010) aseveran que la circunstancia económica, geográfica, cultural y psicológica, influyen en la decisión de compra del consumidor; y es en el interés de la empresa prestar atención a estos factores y tener clara la proporción en la que se relacionan con la situación actual que enfrenta el negocio. 
Figura 1. Modelo de toma de decisiones del consumidor

INFLUENCIAS EXTERNAS

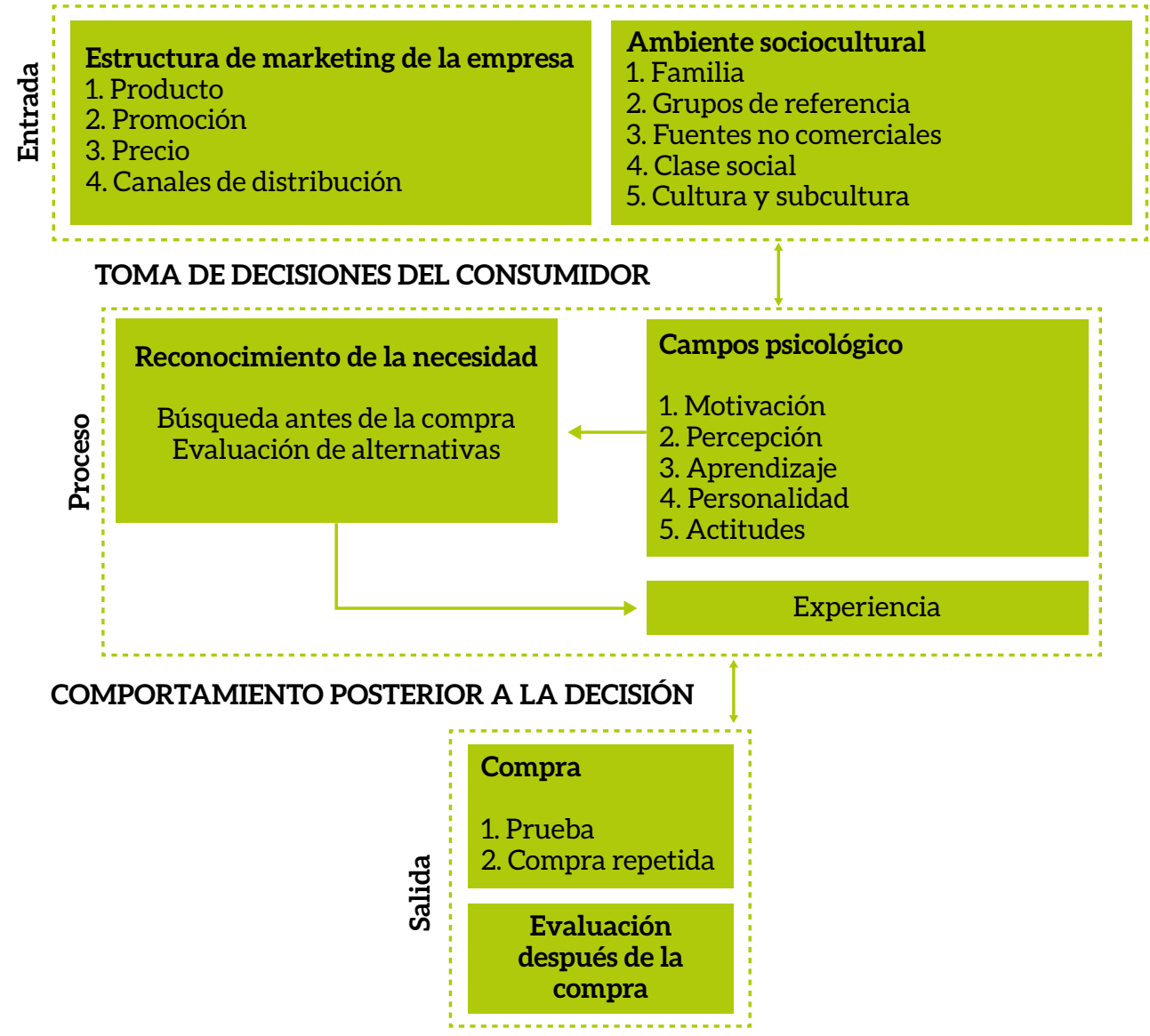

Fuente: Schiffman, Lazar y Wisenblit (2010).

\subsection{Factores que inciden en la decisión de compra del consumidor}

De acuerdo a Solomon (2017) existen tres factores que influyen en el proceso de decisión de compra del consumidor: factores culturales, sociales y personales.

\subsubsection{Factores culturales}

El nivel cultural, es el conjunto de conocimientos que por medio del proceso de socialización el individuo adquiere a lo largo de su vida en la escuela, la familia, la religión y otras instituciones. En base a lo aprendido a lo largo de la vida, se consume solo aquello que conocemos y nos gusta. Cuanto mayor es el nivel cultural, se conocen más cosas y en consecuencia, se tienen más opciones para elegir y consumir. Así mismo, la información que se ha generado sobre los beneficios de consumir productos orgánicos es cada vez mayor y ha permeado en un mayor número de consumidores. Esto ha generado un proceso de concientización sobre el riesgo en la salud al ingerir productos con aditivos y conservadores sintéticos. Por lo tanto, se propone:

H1: La conciencia de beneficios del consumo de alimentos orgánicos influye en la disposición a pagar un sobreprecio.

En el caso de las subculturas, la inmigración favorece la mezcla de personas de diferentes nacionalidades, comunidades y religiones. Cada uno de estos grupos tiene sus costumbres, gustos y necesidades que determinarán su comportamiento a la hora de consumir productos. 
Como parte de la globalización, las redes sociales y el internet, y varios medios de comunicación (TV, programas y películas extranjeras), es cada vez más común, conocer diferentes hábitos, estilos y gustos de diferentes poblaciones, por lo que puede resultar atractivo, comprar productos de otros países.

La clase social también es un factor importante de influencia, ya que éste se conforma por grupos relativamente homogéneos, con un estatus y una jerarquía dependiendo su nivel económico, educación, tipo de trabajo, entre otros. Generalmente, dentro de cada clase, sus hábitos de consumo son similares. Los individuos pertenecientes a una clase aspiran a mejorar, de ahí que su comportamiento como consumidor se enfoque en imitar a las personas con un nivel mayor de ingresos.

\subsubsection{Factores sociales}

La familia y grupos a los que pertenece un individuo, así como el rol y estatus que tiene en sus grupos de referencia con los que interactúa, como los amigos, compañeros de trabajo, profesionales o los conocidos en el ámbito religioso; todos ellos ejercen una influencia sobre el comportamiento del consumidor. En la actualidad las necesidades y demandas de consumo se ven afectadas por los distintos comportamientos de cada tipo de familia. El estatus es una fuerza poderosa puesto que se centra en el respeto o aprecio que se le tiene a aquel individuo que goza de un prestigio entre los miembros de un grupo, ejerciendo una influencia en los patrones de consumo.

\subsubsection{Factores personales}

Elementos como la edad, fase del ciclo de vida, ocupación, estilo de vida, situación económica y personalidad, influyen de manera muy importante en la decisión de compra, sobre todo de productos orgánicos. Las características psicológicas, conductuales, emocionales y sociales que componen la estructura dinámica de un individuo, generan diferentes maneras de enfrentar la vida y por lo tanto influyen en la manera de consumir. El estilo de vida comprende aspectos relacionados con la forma de vivir de una persona como son intereses, opiniones y actividades que determinan finalmente en el comportamiento de consumo de los individuos.

De hecho, se han desarrollado diversos estudios donde analizan los factores personales para caracterizar al consumidor orgánico encontrando resultados distintos. De acuerdo a Shaw, McDonagh, Prothero, Schultz y Stanton (2007) el consumidor orgánico se caracteriza principalmente por ser del género femenino, con hijos y en edad adulta; así mismo, resaltan que a pesar de que el consumidor joven está a favor de los productos orgánicos el principal comprador de estos alimentos son las personas de más edad, sobre todo porque tienen mayor capacidad de pagar un sobreprecio por los orgánicos en relación a los consumidores más jóvenes y, tal vez, porque los problemas de salud son mayores, debiendo cuidar la calidad de su alimentación. En este sentido se plantea la segunda hipótesis de investigación:

H2: La edad de los consumidores influye en la disposición a pagar un sobreprecio por alimentos orgánicos

El nivel educativo también es un factor personal que ha sido estudiado para determinar la disposición a comprar productos orgánicos. Este influye en la capacidad de analizar, sintetizar, indagar y concluir sobre situaciones que se presenten en el ámbito personal o laboral. Cuando el consumidor posee un mayor nivel educativo logra mayor apertura hacia el aprendizaje, incrementando su nivel de conocimiento y sus habilidades para aplicarlo en cualquier ámbito. En este sentido, se espera que tengan un mayor acervo cultural en relación al impacto que tiene en la salud el consumo de productos más limpios. De acuerdo a la OCDE el nivel educativo está relacionado positivamente con la esperanza de vida, lo que implica, por ejemplo, que una persona con estudios de licenciatura vivirá más años en comparación con alguien que posee estudios de nivel básico. Esto significa que el nivel educativo influye en el cuidado de la salud, tal como lo expone (Gómez, 2013) al decir que los consumidores con mayor nivel educativo 
tienen menor probabilidad de padecer obesidad, poseen mejor salud física y participan de manera más activa en programas preventivos de la salud. Al respecto, la tercera hipótesis se plantea de la siguiente manera:

H3: La escolaridad de los consumidores influye en la disposición a pagar un sobreprecio por alimentos orgánicos.

Debido a que el precio de los alimentos orgánicos es considerablemente superior a los alimentos tradicionales, es de esperarse que la situación económica del consumidor ejerza una influencia en la demanda de los orgánicos. Un mayor poder adquisitivo permite destinar un mayor porcentaje del gasto en alimentos orgánicos ya que no representa una restricción financiera. De acuerdo a Frydlová y Vostrá (2011) las principales barreras que existen para el consumo de orgánicos es el sobre precio y el ingreso de los consumidores. Por lo tanto, es importante determinar si el consumo de los orgánicos es exclusivo de un estrato social o depende más bien de otros factores como la salud. De acuerdo a los argumentos anteriores, se propone la siguiente hipótesis:

H4: El nivel socio económico de los consumidores influye en la disposición a pagar un sobreprecio por alimentos orgánicos.

Tal como lo proponen Schiffman, et al. (2010), los esfuerzos de marketing son factores que inciden en el proceso de decisión del consumidor, al igual que los socioculturales y psicológicos que se describieron con anterioridad. En este sentido, las características de los canales de distribución representan un elemento fundamental para incentivar la compra de productos orgánicos, sobre todo en lo que respecta a la distancia, el surtido y el tiempo de traslado. Hino (2010) encontró que la elección del establecimiento para realizar las compras dependerá de la distancia a recorrer y la probabilidad de localizar productos perecederos. Con base en los hallazgos de Melchor y Lerma (2016), los criterios de elección de una tienda son la cercanía de la tienda de su casa (71\%), facilidades de pago (61\%) y la frescura de los productos (13\%). Por lo tanto, es importante para los consumidores potenciales, identificar en dónde pueden conseguir los productos alimenticios que les interesan. De acuerdo con los argumentos anteriores, se plantea la siguiente hipótesis:

H5: La localización de la plaza, influye en la disposición a pagar un sobreprecio por alimentos orgánicos.

A continuación, se presenta el modelo propuesto sobre los factores que influyen en la disposición a pagar un sobreprecio por alimentos orgánicos (Figura 2), los cuales deben ser considerados para determinar el entorno del mercado y facilitar la identificación del perfil de los consumidores potenciales que demandan este tipo particular de alimentos cuyos precios generalmente son más altos que los alimentos tradicionales.

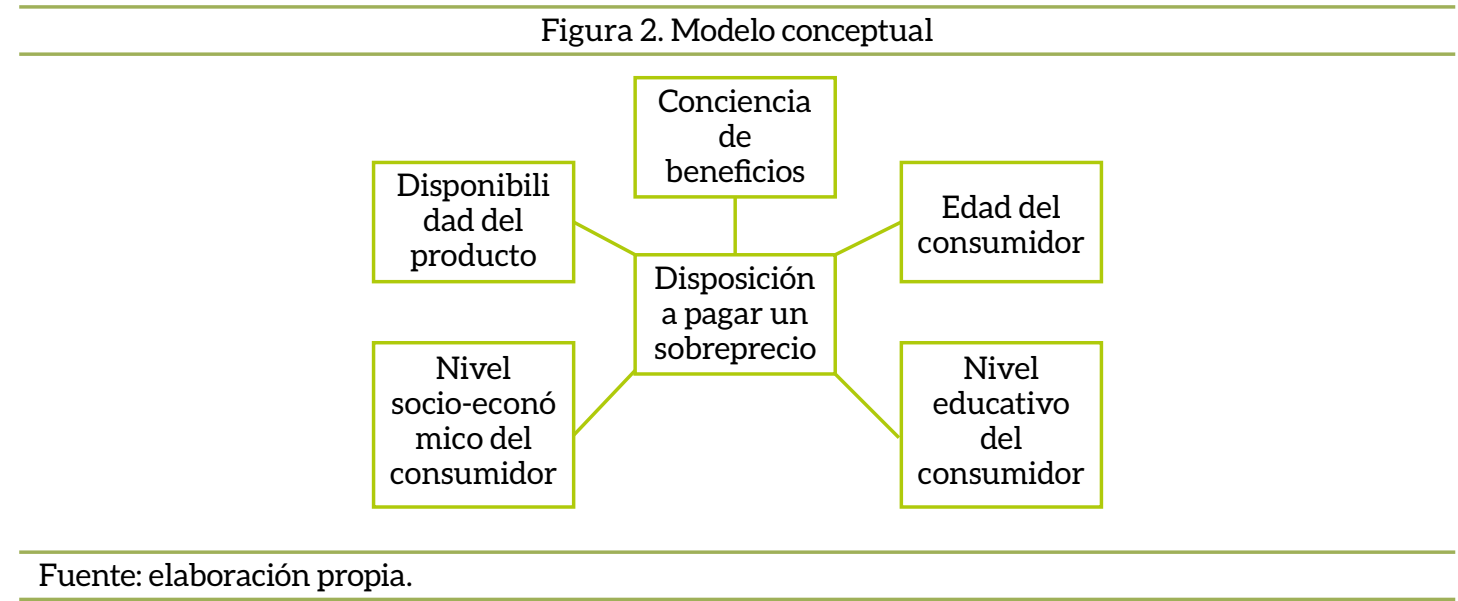




\section{Metodología}

Para lograr los objetivos planteados se llevó a cabo una investigación mixta. La parte cuantitativa de la investigación tiene como objetivo identificar los factores sociales y personales que influyen en la disposición a pagar un sobreprecio por productos orgánicos para lo cual se realizó un análisis de regresión. La partecualitativa se desarrollóa través de sesiones de focus group para analizar de manera más detallada los beneficios, deseventajas, preferencias, frecuencias de consumo, disponibilidad y comprensión del concepto de alimentos orgánicos, desde la percepción del consumidor final.

\subsection{Investigación cuantitativa}

Para llevar a cabo la investigación cuantitativa se diseñó una encuesta con cuatro secciones. En la primera se incluyen datos demográficos del consumidor. En la segunda sección se recaba información sobre la postura en relación a la alimentación sana, preferencias de consumo alimentario, salud, plaza, oferta de alimentos en la región, criterio de compra y disposición a pagar sobreprecio por preferir el consumo de alimentos orgánicos. La medición se hizo en escala Likert de cuatro niveles (1= total desacuerdo, 4= totalmente de acuerdo). La tercera sección incluye preguntas abiertas sobre la disponibilidad de productos orgánicos en la región, las plazas donde se oferta y el periodo de tiempo desde que iniciaron a consumirlos. En la última parte se detectan preferencias por tipo de alimentos y categorías (ver anexo 1).

Para evaluar la pertinencia del instrumento se realizó una prueba piloto, la cual se aplicóa 70 personas elegidas de manera aleatoria y distribuidas en las diferentes colonias de la ciudad para identificar el nivel socioeconómico de los participantes (apoyado en una base de datos avalada por la AMAI). Con base en los resultados de la prueba se realizaron diversos cambios como fueron: a) eliminar algunas preguntas, b) acotar el rango de edades, y c) delimitar el nivel socieconómico. Además, se aplicaron pruebas de confiabilidad y validez para depurar el instrumento y hacerlo más confiable, obteniendo un alpha de cronbach de 686 .

En base a la aplicación de la fórmula de población finita para proporciones, se calculó la muestra probabilística estratificada mediante un nivel de confianza del $95 \%$ y un margen de error de medición del 5\%. El tamaño de la muestra fue de 270 personas, residentes en la ciudad de Celaya, Guanajuato, México, las cuales fueron encuestadas en el primer semestre del 2018.

La muestra está conformada principalmente por consumidores jóvenes adultos que cuentan con al menos educación media superior y pertenecen a alguno de los tres niveles socioeconómicos más privilegiados en México: medio (C), medio alto ( $\mathrm{C}+$ ) y alto (A/B). La Tabla 2 muestra las características específicas de la muestra.

Tabla 2. Características de la muestra

\begin{tabular}{ll} 
Nivel socioeconómico & \\
\hline Medio & $54 \%$ \\
Medio alto & $21.6 \%$ \\
Alto & $20 \%$ \\
\hline Escolaridad & \\
\hline Educación media superior & $33.4 \%$ \\
Educación superior & $42.6 \%$ \\
Posgrado & $6 \%$ \\
\hline Edad & \\
\hline$<25$ años & $44 \%$ \\
$25-40$ años & $30 \%$ \\
$41-64$ años & $25 \%$ \\
$>64$ años & $1 \%$ \\
\hline Género & \\
\hline Mujeres & $53 \%$ \\
Hombres & $47 \%$
\end{tabular}

Fuente: elaboración propia con base en resultados de SPSS. 


\subsection{Investigación cualitativa}

Para llevar a cabo la investigación cualitativa se realizaron cuatro sesiones grupales cada una con doce participantes. Las características que describen el segmento de la muestra son: personas económicamente activas que privilegian un estilo de vida saludable, cuya edad oscila entre los 25 y 60 años, y pertenecen a uno de los tres niveles económicos más altos de México (C, C + y A/B). Dentro de la dinámica en las sesiones de grupo, se incluyeron tres actividades que involucran la aplicación de técnicas proyectivas, como la asociación de palabras.

\section{Resultados}

El análisis descriptivo de los datos muestra que existe un bajo nivel de consumo de alimentos orgánicos en la ciudad de Celaya, México ya que solo el $29 \%$ de las personas los compran. Así mismo, los resultados señalan que la frecuencia de consumo de productos orgánicos es baja ya que el $53 \%$ de los encuestados los adquieren de manera esporádica y sólo el $22.7 \%$ afirma que los alimentos orgánicos forman parte de su estilo alimenticio. También se encontró que la tendencia hacia la alimentación orgánica es relativamente nueva, pues únicamente el 7\% de los usuarios tiene más de cinco años consumiendo, y el 11.5\% tiene menos de dos años con este nuevo plan alimenticio.

Los alimentos orgánicos con mayor demanda son la miel, salsas, queso, té, pescado, carne y bebidas. Las personas consumen productos orgánicos debido a que reconocen los beneficios que representan para la salud y por supuesto porque tienen la capacidad económica para comprarlos. En este sentido, el $89 \%$ de los encuestados están conscientes de los beneficios que tienen para la salud los alimentos orgánicos, sin embargo, dos terceras partes de los encuestados expresaron su intención de comprar productos orgánicos siempre y cuando no exista diferencia en el precio de los productos en comparación con los tradicionales. En específico, el $70 \%$ de las personas están dispuestas a pagar un sobreprecio del $5 \%$ por conseguir alimentos orgánicos; pero si la diferencia de precio es del 10\%, solo el $50 \%$ de los consumidores los comprarían; mientras que si el aumento es del 15\%, solo el 21\% estaría dispuesto a pagar el excedente por conseguir alimentos más saludables.

La mayoría de los consumidores (89\%) están concientes de los beneficios que tienen los alimentos orgánicos, especialmente los relacionados con la salud y con la calidad, siendo este último el principal criterio que las personas utilizan para seleccionar sus alimentos (79.65\%). Sin embargo, poco más de dos terceras partes de los consumidores coinciden en la dificultad que enfrentan para comprar productos orgánicos debido a la oferta tan limitada que existe. Por lo tanto, cuando tienen la intención de conseguir alimentos orgánicos, tienen que viajar a Querétaro, como primera opción, y en segundo lugar San Miguel de Allende.

\subsection{Resultados de grupos focales}

El consumidor de alimentos orgánicos asocia la palabra "orgánico" con saludable, natural, libre de toxinas y agricultura. En este sentido, la mayoría de los participantes concuerdan que los alimentos orgánicos son aquellos que no han sido alterados, que están relacionados con el proceso natural vegetal o animal de los productos, para los cuales no se utilizó un pesticida agroquímico durante su producción.

Entre los productos orgánicos con mayor demanda se encuentra el huevo, vegetales, piloncillo, aceite de coco, avena y carne. Los atributos que toman en cuenta para decidir sobre la compra de un producto alimenticio, son, en orden de importancia: empaque, calidad, color del producto, que sea natural, certificaciones, marca, precio, textura, frescura, origen, sabor, servicio en la tienda, aroma, caducidad, fecha, ingredientes, y finalmente, información nutrimental.

\subsubsection{Razones para consumir alimentos orgánicos}

Se identificaron tres razones por las cuales las personas manifiestan especial interés en consumir alimentos orgánicos: 1) cuidado de la salud, 2) mayor calidad de los alimentos y 3) 
Figura 3. Beneficios particulares que impactan la salud

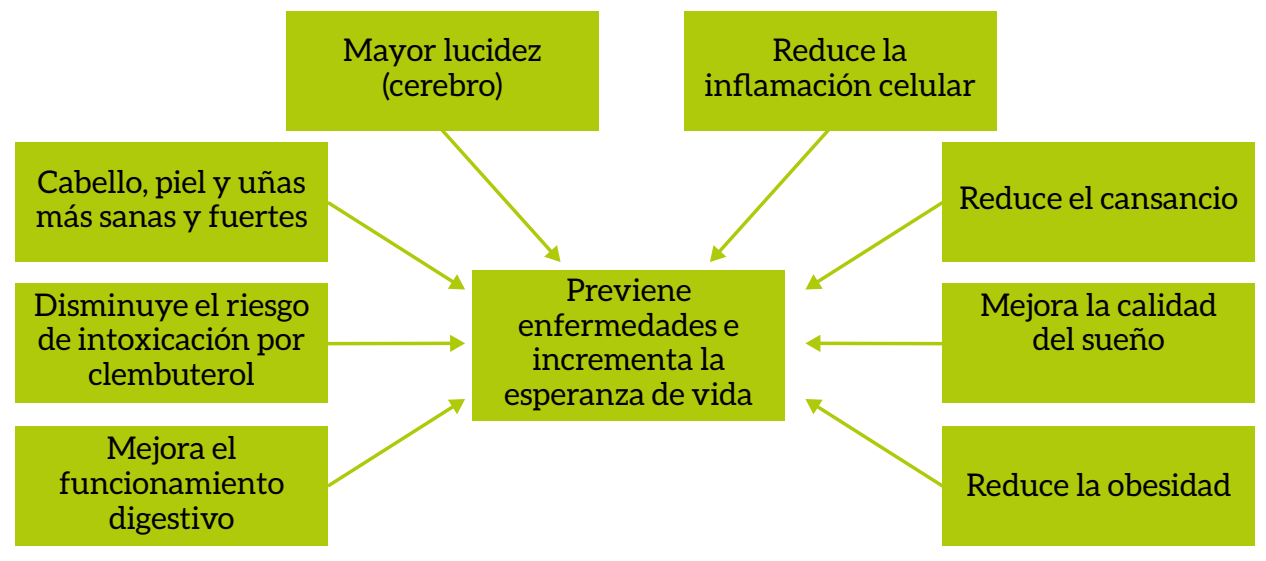

Fuente: elaboración propia.

protección del medioambiente. En relación al cuidado de la salud, los participantes identifican diversos beneficios cuando se consumen productos orgánicos los cuales se muestran en la Figura 3.

Así mismo, están convencidos del surgimiento de enfermedades a partir del consumo de alimentos procesados e industrializados. Al respecto, la mayoría de los participantes consideran que la principal consecuencia de consumir alimentos industrializados que contienen químicos es la enfermedad del cáncer, el desarrollo de alergias y la aceleración del proceso hormonal y menstrual de las niñas (debido a la inyección de hormonas a pollos y vacas para fomentar su rápido desarrollo y crecimiento). Incluso consideran el consumo de alimentos orgánicos como medida preventiva a diversos padecimientos de salud.

En cuanto a la calidad de los alimentos hacen referencia a un mejor sabor de los alimentos orgánicos, ya que al no contener químicos ni sabores artificiales, el paladar detecta un sabor más natural y real, por la concentración del sabor. Diversos testimonios refieren una notoria diferencia entre un producto lácteo proveniente de una vaca tratada con hormonas, en comparación con las de libre pastoreo. También identifican en los alimentos orgánicos una mejor textura (por ejemplo, frutas y verduras más crujientes), así como una diferenciación en olor y color, sobre todo en alimentos como la carne y el huevo. Todas estas características diferenciadoras las atribuyen a la ausencia de conservadores y residuos por herbicidas e insecticidas. Sin embargo, señalan que en la mayoría de los casos, los alimentos orgánicos tienen un periodo de vida mucho más corto.

La protección al medio ambiente también es una razón importante para algunos consumidores orgánicos ya que evitan, en la medida de lo posible, el sufrimiento de animales, mantienen la fertilidad de la tierra y contaminan menos el ambiente.

El primer contacto que tuvieron con los alimentos orgánicos se debe a la experiencia que obtuvieron de vivir en el extranjero, específicamente en países desarrollados, donde esta forma de alimentación se vuelve cada vez más común, encontrando una gran oferta de alimentos orgánicos. Otra razón por la cual se inician como consumidores orgánicos es la vivencia cercana de problemas de salud ya sean propios, de conocidos o familiares.

Por otro lado, también existe la percepción de que los productos orgánicos se han convertido en una tendencia y son más bien moda. Una nutrióloga comenta que las empresas sólo usan el término "orgánico" para vender el producto más caro, y pone en duda que los productos de los supermercados sean orgánicos; de acuerdo con su experiencia, si un producto procesado tiene más de 5 ingredientes, no es orgánico, ni saludable por lo que sugiere tomar en cuenta las etiquetas para valorarlo. De hecho, el $50 \%$ de los participantes revisan las etiquetas de los 
productos que consumen. Otros consumidores orgánicos no confían por completo en el valor nutricional, calorías y porciones que las empresas publican en sus etiquetas, pues consideran que la información del empaque es engañoso. Estas apreciaciones muestran la divergencia de opinión entre consumidores sobre la conveniencia de comprar productos orgánicos al valorar el costobeneficio.

\subsubsection{Barreras al consumo de alimentos orgánicos}

Existen diversas razones por las cuales resulta difícil consumir estos productos. La principal de ellas es el precio tan alto que se paga por adquirir alimentos orgánicos certificados. Quienes valoran su calidad están conscientes del costo de producción debido a la inocuidad y la certificación. En este sentido, el 95\% de los participantes de las sesiones de grupo están de acuerdo con que la alimentación es la base para evitar el cáncer y están dispuestos a pagar un sobre precio por cuidar su salud. De hecho, cada vez más personas están optando por cultivar sus propias frutas y verduras (30\% de los participantes conocen gente que tienen sus propios cultivos).

Otra barrera al consumo de orgánicos se debe a la limitada disponibilidad de este tipo de productos. En la ciudad de Celaya, los participantes identificaron ocho lugares donde pueden adquirir alimentos orgánicos (ver Tabla 3). Los productos disponibles en estas tiendas son: vegetales, lácteos, barras de cereal, congelados (pescado / nuggets para los niños), granos ancestrales (arroz, quinoa, avena, amaranto, arroz integral, arroz negro), tofu, queso, yogur griego, y aceites de uva, aguacate y oliva.

Sin embargo, muchos consumidores manifiestan la necesidad de trasladarse a otras ciudades cercanas como Querétaro o San Miguel de Allende para comprar alimentos menos comunes y/o conseguir marcas específicas de productos (Certificado Kosher), por la confianza que les brinda en cuanto a calidad y garantía de ser un producto limpio. Por otro lado, existen consumidores que buscan, en pequeños municipios y ranchos, productos no industrializados a un precio más accesible que los alimentos con certificación orgánica. Por ejemplo, en el municipio de Apaseo adquieran el jitomate, verduras y blanquillos de gallinas de rancho. Algunos otros mencionan un rancho dentro del municipio de Celaya donde consiguen nopales, tortillas, elotes y el maíz.

La oferta tan limitada que existe en la ciudad se debe también a que la mayoría de los alimentos orgánicos se destinan a la exportación pues son productos que se venden a un precio más alto en relación al que puede pagar un consumidor local; además, el nivel de demanda aún no es suficiente para hacer crecer el mercado e incrementar la oferta de productos.

La corta vida en anaquel del alimento orgánico, la dificultad de comprobar que los productos son en realidad orgánicos y la vida social son otras barreras a la demanda de estos productos. En promedio, el $50 \%$ de los alimentos que consumen los encuestados son de origen orgánico, ya que a veces comen en la calle y en restaurantes.

Por otro lado, la indiferencia de la mayoría de la población hacia lo orgánico se debe a: 1) la desinformación sobre los daños que causan a la salud los productos industrializados; 2) la influencia social sobre una alimentación poco saludable y de tradición en nuestra cultura; 3) el estilo de vida que no privilegia la salud; y 4) el ambiente laboral.

\subsection{Resultados del análisis de regresión}

El análisis de las variables que influyen en la disposición a pagar un sobreprecio por productos orgánicos, se presenta en la Tabla 4. En el modelo 1, la regresión se calcula con una disposición

Tabla 3. Tiendas de productos orgánicos en Celaya, Guanajuato

\begin{tabular}{cccc}
\hline TRESCUARTOS & Costco & Ranchitos & Biotienda Orgánica \\
\cline { 2 - 4 } Royal(Alameda) & Tiendita en Av. Las fuentes & Walmart & Querétaro \\
\hline San Miguel de Allende & Lakshmi(tienda vegetariana) & Apaseo & La Berenjena \\
\hline Fuente. elaboración propia con base en testimonios. & & \\
\hline
\end{tabular}




\begin{tabular}{|c|c|c|c|}
\hline \multicolumn{4}{|c|}{ Tabla 4. Análisis de Regresión Múltiple } \\
\hline Variables & Model 1 & Model 2 & Modelo 3 \\
\hline Constante & $2.621^{* * *}$ & $1.319^{* * *}$ & $0.772^{* * *}$ \\
\hline Nivel socioeconómico(NSE) & -0.081 & -0.029 & -0.007 \\
\hline Escolaridad & -0.060 & -0.082 & -0.046 \\
\hline Edad & 0.089 & $0.163^{* *}$ & $0.180^{* * *}$ \\
\hline Conciencia de beneficios & $0.144^{* *}$ & $0.272^{* * *}$ & 0.071 \\
\hline Localización & -0.045 & $0.159^{* *}$ & $0.363^{* * *}$ \\
\hline F-value & $4.316^{* *}$ & $12.313^{* * *}$ & $18.499^{* * *}$ \\
\hline $\mathrm{R} 2$ & .021 & .163 & .168 \\
\hline
\end{tabular}

Fuente: elaboración propia con base en resultados de SPSS.

a pagar un sobreprecio del $5 \%$. Los resultados revelan que el modelo es significativo $(p<.05)$ y explica el $2.1 \%$ de la varianza en la disposición a pagar un sobreprecio del $5 \%$. En este modelo solamente la conciencia de beneficios influye de manera positiva y significativa en la decisión de comprar alimentos con mayor valor económico ( $\mathrm{p}<.05)$.

En el segundo modelo la variable dependiente está representada por la disposición a pagar un sobreprecio del 10\%. Los resultados indican que el modelo también es significativo ( $p<.01$ ) con una capacidad explicativa del 16.3\%, la cual es notablemente superior al modelo anterior. No obstante, la capacidad explicativa aún es baja. En específico, la edad, la localización y la conciencia de beneficios son variables positivas y significativas que determinan la disposición a pagar un sobreprecio del 10\% por alimentos orgánicos.

El tercer modelo muestra las variables que influyen en la disposición del cliente a pagar un sobreprecio del $15 \%$ por productos orgánicos. Al respecto, la localización y la edad siguen siendo variables positivas y significativas, sin embargo, la conciencia de beneficios ya no resulta significativa en este modelo. Prácticamente no existen diferencias en la capacidad explicativa del segundo y tercer modelo (16.8\%).

Con los resultados expuestos en los tres modelos de regresión podemos determinar que la hipótesis 1 se acepta parcialmente al encontrar que la conciencia de beneficios en relación al consumo de alimentos orgánicos influye en la disposición a pagar un sobreprecio de hasta un $10 \%$.

La influencia que ejerce la edad en la compra de productos orgánicos con un sobreprecio se evalúa en la segunda hipótesis. De acuerdo al segundo y tercer modelo, la edad del consumidor es una variable significativa ( $\mathrm{p}<.05, \mathrm{p}<.01$, respectivamente) lo que implica que la etapa de vida del cliente determina la inclinación a pagar un sobreprecio de hasta $15 \%$ por alimentos orgánicos. Debido a que el coeficiente de la variable edad es positivo, se deduce que a mayor edad del cliente mayor disposición a pagar un sobreprecio por alimentos orgánicos. En este sentido, y debido a que los beneficios en la salud es una de las principales razones por las cuales los consumidores prefieren productos orgánicos, es razonable identificar a las personas de mayor edad con problemas más latentes de salud que los más jóvenes, siendo esto un elemento central para su consumo. Por lo anterior, se acepta la hipótesis dos.

En relación a la tercera hipótesis, la escolaridad tampoco resulta significativa en ningún modelo. Se espera que a mayor nivel educativo las personas desarrollen hábitos más saludables por la información que tienen a su alcance sobre el tema, y están más conscientes de la importancia del cuidado de la salud. No obstante, los resultados muestran que no existe relación entre el nivel educativo y la disposición a pagar un sobreprecio por productos orgánicos, por lo que no se acepta la tercera hipótesis.

La hipótesis cuatro no se acepta, ya que el nivel socio económico de los consumidores no resultó ser significativo en ninguno de los tres modelos, lo cual implica que la capacidad económica 
de las personas no determina el consumo de productos orgánicos, destacando la importancia de otros factores no económicos como lo apunta la literatura. Lo que es importante subrayar es que el coeficiente de la variable de nivel socioeconómico es negativo en los tres modelos, y aunque no es significativo, esto indicaría que a mayor nivel socioeconómico menor es la tendencia a pagar un sobreprecio por productos orgánicos.

La disponibilidad del producto también es una variable significativa en la decisión de compra de alimentos orgánicos con un precio superior de hasta $15 \%(p<.01)$, por lo que se acepta la hipótesis cinco. La intensidad de la relación entre la variable localización y la disposición a pagar un sobreprecio es mayor en el tercer modelo, lo que implica que cuando las personas están dispuestas a pagar un precio mayor al de los productos tradicionales, la disposición de los productos es un factor que el cliente valora y por lo cual están dispuestos a pagar más simplemente por su disponibilidad.

\section{Conclusiones}

El objetivo de este estudio fue analizar el mercado y los factores que determinan la disposición a pagar un sobreprecio por alimentos orgánicos. Los resultados muestran que la conciencia de beneficios, la edad del consumidor y la disponibilidad del producto son variables que explican la disposición a pagar un mayor precio por alimentos orgánicos. Esto implica que las personas que conocen las diversas ventajas que conlleva consumir productos orgánicos tienen mayor probabilidad de invertir en estos alimentos. Así mismo, la edad resulta ser un factor determinante del consumo de los orgánicos debido a los problemas de salud que enfrentan a una edad más adulta, lo cual está relacionada con el hecho de ser la salud la principal razón por la cual se consumen alimentos orgánicos. Debido a que el mercado de los orgánicos aún está en su etapa de crecimiento todavía no se ha logrado generar la oferta suficiente de alimentos limpios por lo cual es difícil encontrar variedad de orgánicos. Se esperaría que el nivel socio económico y el nivel de estudios ejercieran una influencia importante en el consumo de alimentos orgánicos debido al sobreprecio al cual se adquieren. No obstante, los resultados en este estudio muestran que el nivel socioeconómico y el nivel educativo no están relacionados con la probabilidad de comprar alimentos orgánicos, coincidiendo con los hallazgos de otros estudios (Vecchio, Van Loo y Annunziata, 2016; Durham, 2007). Y aunque el consumidor expresa que el precio es la principal limitante para consumir los productos orgánicos, la baja asociación del nivel socioeconómico con la compra de alimentos orgánicos indica que existen otros factores que los consumidores valoran al momento de decidir la compra de alimentos.

Los resultados obtenidos tienen diversas implicaciones. Los consumidores deben modificar sus patrones de consumo donde tomen en cuenta las implicaciones ecológicas y sociales de su consumo y no solamente el factor económico. Esto significa que se debe tener más consciencia de las externalidades positivas que se generan con el consumo de alimentos orgánicos y cómo su consumo impacta en el medioambiente, en la economía de la región, en el apoyo a los pequeños productores y a la salud. Las empresas deben desarrollar tecnología que permita aumentar la vida comercial de los orgánicos y disminuir costos de producción para eliminar la desventaja en su precio. El sector gubernamental debe desarrollar políticas públicas que incentiven la producción de alimentos orgánicos, la innovación de nuevos sistemas de producción orgánica, la incorporación de pequeños productores a la producción orgánica y sobre todo, a generar conciencia de los beneficios en la salud, en la economía y en el medioambiente que conlleva el consumir alimentos orgánicos. 


\section{ANEXO 1. PREGUNTAS DEL CUESTIONARIO}

1. Considero que soy una persona que cuida la calidad de los alimentos que ingiero

2. Estoy consciente de que los alimentos que elijo para cada comida del día, tienen una consecuencia en mi salud

3. Conozco los beneficios que trae a mi salud, el consumo de productos orgánicos

4. Identifico 3 tiendas donde venden productos orgánicos aquí en Celaya

5. En Celaya hay tiendas donde encuentro todos los productos orgánicos que me gusta consumir

6. En general, el grupo más cercano que influencia mis decisiones de compra, por recomendaciones son:

Amigos b. Familia c. Pareja d. Medios (programas tv, artículos de revistas, programas de radio) e. Influencers ( blogs, canales de youtube, etc).

7. Estoy consciente de que el pollo orgánico es mejor para mi salud ya que no está alterado con hormonas

8. Estoy consciente de que la carne orgánica es mejor para mi salud ya que no está alterada con hormonas y químicos como clenbuterol

9. Estoy consciente de que los vegetales orgánicos son mejores para mi salud ya que están libres de pesticidas dañinos para mi organismo

10. Mi criterio de compra de un producto alimenticio es en primer lugar la calidad

11. Estoy dispuesto a pagar un sobreprecio por preferir un producto orgánico (alimento ecológico producido con procedimientos libres de pesticidas, herbicidas y fertilizantes artificiales que dañan la salud)
a. De hasta un $5 \%$
b. De hasta de un $10 \%$
c. De hasta de un $15 \%$

12. Marca con una X en la columna de "si "o "no", dependiendo por cuál producto sí estarías dispuesto a pagar un sobreprecio para consumirlo

13. Yo sustituiría mi compra (productos alimenticios), por un alimento orgánico siempre y cuando el precio fuera el mismo

14. ¿Qué productos orgánicos has buscado en Celaya y no has encontrado ??

15. Si no encontraste el producto orgánico que necesitas, cuáles han sido tus otras opciones de tiendas (donde vendan productos orgánicos) para conseguirlos ( puede ser fuera de Celaya, como Qro. o san Miguel de Allende)

16. ¿Desde hace cuanto tiempo consume productos orgánicos y porqué comenzó a consumirlos? 


\section{Referencias}

Amador, L. (2005). Productos orgánicos retorno a lo natural. PROFECO. 58-62. Recuperado de https:// www.profeco.gob.mx/revista/publicaciones/adelantos_05/organicos_oct05.pdf

Durham, C. (2007). The impact of environmental and health motivations on the organic share of purchases. Agricultural and Resource Economics Review, 36 (2), 304-320. https://doi.org/10.1017/ S1068280500007115

Frydlová, M. y Vostrá, H. (2011). Determinants influencing consumer behaviour in organic food market. Acta Universitatis Agriculturae et Silviculturae Mendelianae Brunensis, LIX (7), 111- 120. Recovered https://acta.mendelu.cz/media/pdf/actaun_2011059070111.pdf

Global Organic Trade Guide (2017). Organic Mexico. Revisado en https://globalorganictrade.com/country/ mexico

Gómez, T. (2013). Beneficios de la educacion superior para el individuo y la sociedad: perspectiva económica y no económica. Research Institute for Higher Education and Science, en http://www.inaecu. com/beneficios-de-la-educacion-superior-para-el-individuo-y-la-sociedad-perspectiva-economica-yno-economica/

Gómez, M., Gómez, L. y Schwentesius, R. (2002). Dinámica del mercado internacional de productos orgánicos y las perspectivas para México. Momento Económico, (120), 54-68. Recuperado de http:// revistas.unam.mx/index.php/rme/article/view/4304/3837

González, E. (2017). La agricultura orgánica en México. En https://www.mexicampo.com.mx/la-agriculturaorganica-en-mexico-2/

Higuchi, A. (2015). Características de los consumidores de productos orgánicos y expansion de su oferta en Lima. Apuntes, XLII, (77), 57-89. Recuperado http://www.scielo.org.pe/scielo.php?script=sci arttext\&pid=S0252-18652015000200002

Hino, H. (2010). Antecedents of supermarket formats' adoption and usage: A study in the context of nonwestern customers. Journal of Retailing and Consumer Services, 17(1), 61-72. https://doi.org/10.1016/j. jretconser.2009.09.005

Lamb, C.W., Hair, J. F. Jr., \& McDaniel, C. (2011). Marketing (11 ed). London, UK: CENGAGE Learning.

Melchor, M. y Lerma, C. (2016). Decisión de compra en tiendas y supermercados: evidencias de consumidores por estratos en Cali-Colombia. Revista Global de Negocios, 4 (2), 97-107. Recovered https:// papers.ssrn.com/sol3/papers.cfm?abstract_id $=2665375$

Organización de las Naciones Unidas para la Alimentación y la Agricultura (ONNUA). (2019). ¿Qué es la agricultura orgánica? Recuperad en http://www.fao.org/organicag/oa-faq/oa-faq1/es/

Procuraduría Federal del Consumidor (PROFECO) (2018). Alimentos orgánicos. Recuperado https://www. gob.mx/profeco/documentos/alimentos-organicos?state=published

Proméxico (2017). Alimentos orgánicos en México. Unidad de Inteligencia de Negocios. Recuperado http:// www.promexico.gob.mx/template/ciie/docs/sectores/alimentos-organicos-en-mexico.pdf

Ramírez, M. (2016). El mercado de productos orgánicos crece 10\% cada año. Milenio. Recuperado http:// www.milenio.com/negocios/productos_organicos-veganos-vegetarianos-comer_sano_0_708529215. html

Schiffman, L. G., Kanuk, L. L., \& Wisenblit, J. (2010). Modelo de toma de decisiones del consumidor. Comportamiento del consumidor. México D.F., México. Prentice Hall.

Shaw, R., McDonagh, P., Prothero, A., Schultz, C. y Stanton, J. (2007). Who are organic food consumers? A compilation and review of why people purchase organic food. Journal of Consumer Behaviour, 6, 94110. https://doi.org/10.1002/cb.210 
Schifferstein, H. y Ophuis, O. (1998). Health-related determinants of organic food consumption in the Netherlands. Food Quality and Preference, 9 (3), 119-133. https://doi.org/10.1016/S0950-3293(97)00044-X

Schwentesius, R., Gómez, M, Ortigoza, J. y Gómez, L. (2014). México Orgánico. Situación y Perspectivas. Agroecología, 9 (1 y 2), 7-15. Recuperado de https://dialnet.unirioja.es/servlet/articulo?codigo=6062887

Solomon, M.R., (2017). Comportamiento del consumidor (11 ed.). Naucalpan de Juárez, México: Pearson Educación..

Viera, D., Gálvez, D., y Navarro, I. (2010). Factores que influyen en la decisión de compra de Barrio- el caso de la ciudad de Arica-Chile. Horizontes Empresariales, 9(1), 29-46. Recuperado http://revistas.ubiobio.cl/ index.php/HHEE/article/view/2035

Vecchio, R., Van Loo, E.J., Annunziata, A. (2016). Consumers' willingness to pay for conventional, organic and functional yogurt: evidence from experimental auctions. International Journal of Consumer Studies, 40 (3), 368-378. https://doi.org/10.1111/ijcs.12264

Willer, H., Lernoud, J. y Kemper, L. (2018). The word of organic agriculture 2018: Summary. FiBL \& IFOAM - Organics International. Suecia. https://www.organic-world.net/yearbook/yearbook-2018/pdf.html

Zielke, S. (2010). How price image dimensions influence shopping intentions for different store formats. European Journal of Marketing, 44 (6), 748-770. https://doi.org/10.1108/03090561011032702 